\section{Emphasis on Preventing Microscopic Positive Margins in Gastric Cancer}

\section{TO THE EDITORS:}

In the November issue of Annals of Surgical Oncology, Sun et al. report the results of their large retrospective study on the impact of positive resection margins on outcomes of patients with gastric cancer. ${ }^{1}$

The crucial role of quality of surgery in achieving complete tumor resection (R0) in patients with potentially curable gastric cancer has been widely recognized., ${ }^{2,3}$ Especially in less advanced pT1-2, pN0-1, and I-II stage, with good patient prognosis after adequate R0, D2 surgery, presence of cancer cells at resection margins may reverse the excellent outcome, leading to local recurrence and death. By contrast, in more advanced pT3-4, pN2-3, and III-VI stage with overall poor survival, microscopic margin status may have less prognostic value.

Sun and colleagues have precisely evaluated this issue. ${ }^{1}$ They compared clinicopathologic and survival follow-up data between 110 patients with positive margins and 2,159 patients with negative margins. All patients had undergone gastrectomy with D2/D3 lymphadenectomy. The overall survival rate was significantly better among patients with negative margins than among those patients with positive margins. A subgroup analysis showed significant differences in survival regarding margin status only for patients with pT1-2, pN0-1, and I-II stage, but not among those with pT3-4, pN2-3, and IIIVI stage. The authors conclude that margin status influences survival of patients with early stage I-II after D2/D3 lymphadenectomy, but not for those with advanced stage III-IV.

This study provides very useful information. The results sound logical and expectable. However, based on these data, should we give emphasis to achieving cancer-free resection margins only for early stage I-II but not for advanced stage III?

It is now recommended that every effort for negative margins should be made even for advanced tumor stage pT3-4N1-2 when R0 resection is intended, for two prevailing reasons. Firstly, the number of patients with marginpositive pt3-4N2-3 is small. Many of these patients die from distant metastasis, and thus margin status does not affect survival. However, some patients have localized disease and are at low risk of distant recurrence. In these patients, negative margins are important for long-term survival. Therefore, a much larger number of these patients would be required to reach statistically significant difference.

Secondly, now the addition of trastuzumab to chemotherapy in patients with human epidermal growth factor receptor 2 (HER2)-positive advanced gastric cancers significantly improves survival. ${ }^{4}$

Thus, these patients with both negative margins and trastuzumab treatment may have a good chance for longterm survival. Given that excellent translational research based on genetics and genomics advances promises to provide new effective targeted agents. ${ }^{5}$

Aim of surgery with D2 gastrectomy is to achieve excellent locoregional tumor control. This locoregional recurrence prevention with negative margins even for advanced tumor stage together with modern adjuvant chemotherapy and targeted therapy may improve survival even of patients with advanced, resectable nonmetastatic gastric cancer.

\section{J. Spiliotis, MD}

Department of Surgery, Metaxa Cancer Hospital, Pireaus, Greece

e-mail: jspil@in.gr

Published Online: 21 January 2010

(C) Society of Surgical Oncology 2010

\section{REFERENCES}

1. Sun Z, Li DM, Wang ZN, et al. Prognostic significance of microscopic positive margins for gastric cancer patients with potentially curative resection. Ann Surg Oncol. 2009;16(11):302837.

2. Roukos DH. Genome-wide association studies and aggressive surgery toward individualized prevention, and improved local control and overall survival for gastric cancer. Ann Surg Oncol. 2009;16(4):795-98.

3. Roukos DH, Paraschou P, Lorenz M. Distal gastric cancer and extensive surgery: a new evaluation method based on the study of the status of residual lymph nodes after limited surgery. Ann Surg Oncol. 2000;7(10):719-26.

4. Roukos DH. Targeting gastric cancer with trastuzumab: new clinical practice and innovative developments to overcome resistance. Ann Surg Oncol. 2009 Oct 20. [Epub ahead of print]. doi: 10.1245/s10434-009-0766-0.

5. Roukos DH. Mea culpa with cancer-targeted therapy: new thinking and new agents design for novel, causal networks-based, personalized biomedicine. Expert Rev Mol Diagn. 2009 Apr; 9(3):217-21. 